Hardy-Ramanujan Journal

Vol.25 (2002) 5-21

\title{
SOME PROBLEMS OF ANALYTIC NUMBER THEORY -IV
}

\author{
R.Balasubramanian and K.Ramachandra.
}

1. Introduction. There are two main reasons for publishing this paper. The earlier paper III with the same title was published under many difficulties and consequently in that paper $[R B, K R]$ the pages were not numbered properly. Secondly in the present paper we use Ramachandra's kernel function of the second order (see Lemma 1 of §3) namely $\operatorname{Exp}\left((\sin z)^{2}\right)$. This has some advantages over the earlier kernel $\operatorname{Exp}\left(z^{4 a+2}\right)$ where $a$ is a positive integer. As an outcome of the new kernel we are able to handle $\Omega$-Theorems for error terms in the asymptotic formula for the summatory function of the coefficients of generating functions of the type $\operatorname{Exp}(\zeta(s)), \operatorname{Exp} \operatorname{Exp}(\zeta(s))$ and also of the type $\operatorname{Exp} \operatorname{Exp}\left((\zeta(s))^{\frac{1}{2}}\right)$. (Also there follow many applications such as $L(s, \chi)$ in place of $\zeta(s))$. It will be plain from our Theorems 3 and 4 which follow from Theorem 1 of the present paper especially from Theorems 2 and 3) that $\Omega$ Theorems in divisor problems such as

$$
\sum_{n \leq x} d_{k}(n)-x P_{k}(\log x)=\Omega\left(x^{\frac{1}{2}-\frac{1}{2 k}}\right)
$$

do not need the functional equation (for $\zeta(s)$ ) at all. (Here $(\zeta(s))^{k}=\sum_{n=1}^{\infty} d_{k}(n) n^{-s}($ Res $\geq$ $2), k \geq 2$ is any integer and $P_{k}(\log x)$ is a polynomial of degree $k-1$ in $\left.\log x\right)$. One of the corollories to Theorem 1 is Theorem 2. It gives $\Omega_{ \pm}$theorems (but not with the same exponent as in $\Omega$ theorems). For instance in the case of the abelian group problem we get $\Omega_{ \pm}\left(x^{\frac{1}{10}}\right)$ (or a slightly better result) whereas we get $\Omega\left(x^{\frac{1}{6}} \sqrt{\log x}\right.$ ). Our method $[\mathrm{RB}, \mathrm{KR}]$ of proving these two results is explained beautifully in a joint paper [AS, KS] by A.Sankaranarayanan and K.Srinivas. More complicated version of the same method was employed by R.Balasubramanian, K.Ramachandra and M.V.Subbarao [RB, KR, MVS] to study the oscillation of the error-term in the asymptotic formula for the number of k-full numbers not exceeding $x$. This method has been applied by A.Schinzel [AS] to study a problem considered by Sierpinski and Ramanujan. Here the generating function will have many singularities which are not too densely distributed. In the present paper we formalise this aspect of the problem also. The crux of the whole thing seems to be a fundamental identity given by Theorem 1 from which all the other theorems follow. 


\section{§. NOTATION AND STATEMENT OF THEOREM 1.}

Throughout this paper

$$
F(s)=\sum_{n=1}^{\infty} a_{n} \lambda_{n}^{-s}(s=\sigma+i t)
$$

where $a_{1}, a_{2}, \ldots$ is a sequence of complex numbers, $1=\lambda_{1}<\lambda_{2}<\lambda_{3}, \ldots$ and $\lambda_{n+1}-\lambda_{n} \gg$ 1. It will be assumed that $F(s)$ converges absolutely in some half-plane $\sigma \geq C_{0} \geq 100$ and analytically continuable in $(\sigma \geq \alpha-\delta, t \epsilon I)$ where $\alpha$ and $\delta$ are constants satisfying $\alpha>\delta>0\left(0<\delta<\frac{1}{2}\right)$ (the last requirement is not very important). I is a suitable interval for $t$. For the purposes of Theorems 2 and $3,\left\{a_{n}\right\}$ is any sequence of complex numbers and in certain applications of Theorem 4 we assume that $\left\{a_{n}\right\}$ is any sequence of real numbers and at the same time we assume that $|F(s)|$ does not exceed a bounded (positive constant) power of $|t|$ for all large $|t|$. A will be a sufficiently large constant and for $u>0$ we define

$$
\Delta(u)=\frac{1}{2 \pi i} \int_{C_{0}-i \infty}^{C_{0}+i \infty} u^{w}\left(\operatorname{Exp}\left(\left(\sin \frac{w}{A}\right)^{2}\right)\right) \frac{d w}{w}
$$

and also

$$
J(u)=\frac{1}{2 \pi i} \int_{C_{0}-i \infty}^{C_{0}+i \infty} u^{w}\left(\operatorname{Exp}\left(\left(\sin \frac{w}{A}\right)^{2}\right)\right) d w .
$$

We remark that $\Delta(u)=O\left(u^{A / 2}\right)$ and also $1+O\left(u^{-A / 2}\right)$ and is therefore bounded by a constant depending only on $A$. Similarly $J(u)=O\left(u^{A / 2}\right)$ and also $O\left(u^{-A / 2}\right)$ and is therefore bounded by a constant depending only on $A$. These follow by moving the line of integration to $\operatorname{Re}(w)=A / 2$ and $-A / 2$ respectively. With $F(s)$ we associate the series

$$
B(s)=\sum_{n=1}^{\infty} a_{n} \lambda_{n}^{-s} \Delta\left(\frac{X}{\lambda_{n}}\right)
$$

where $X>0$.

THEOREM 1.Let $s_{0}=\alpha+i t_{0}\left(t_{0}\right.$ is assumed to exceed a large positive constant) and $\max |F(s)| \leq M$ where the maximum is taken over $\left(\sigma \geq \alpha-\delta,\left|t-t_{0}\right| \leq C_{1} \log \log M\right)$ and we impose the condition $t_{0} \leq M \leq \operatorname{Exp} \operatorname{Exp}\left(\varepsilon t_{0}\right)$ where $C_{1}$ is a large positive constant and $\varepsilon$ is a small positive constant. Also we impose $0<\delta<\alpha$. Then fixing $\delta$ to be $A^{-2}$ and $X$ to be $M^{\delta^{-2}}$, we have (by simple contour integration)

$$
F\left(s_{0}\right)=B\left(s_{0}\right)+O\left(M^{-A}\right)
$$

Also if $\phi(z) z^{-1}$ is any analytic function (analytic on a curve $C$ to be described presently) and

$$
E(u)=\sum_{\lambda_{n} \leq u} a_{n}-\frac{1}{2 \pi i} \int_{z \in C} u^{z} \phi(z) \frac{d z}{z}
$$


We have (for any large positive constant $d_{0}$ )

$$
\begin{aligned}
B\left(s_{0}\right) & =\sum_{\lambda_{n} \leq y} a_{n} \lambda_{n}^{-s} \Delta\left(\frac{X}{\lambda_{n}}\right)+B_{1}\left(s_{0}\right) \\
& +s_{0} \int_{y}^{\infty} E(u) u^{-s_{0}-1} \Delta\left(\frac{X}{u}\right) d u+\int_{y}^{\infty} E(u) u^{-s_{0}-1} J\left(\frac{X}{u}\right) d u \\
& +\frac{1}{2 \pi i} \int_{z \epsilon C} \phi(z)\left(\operatorname{Exp}\left(\left(\sin \frac{-s_{0}+z}{A}\right)^{2}\right)\right) \frac{X^{-s_{0}+z}}{-s_{0}+z} d z \\
& -\frac{1}{2 \pi i} \int_{z \epsilon C} \frac{y^{-s_{0}+z}}{-s_{0}+z} \phi(z) d z \\
- & \frac{1}{2 \pi i} \int_{z \epsilon C} \phi(z)\left(\frac{1}{2 \pi i} \int_{-d_{0}-i \infty}^{-d_{0}+i \infty} \frac{y^{-s_{0}+z}}{-s_{0}+z-w}\left(\frac{X}{y}\right)^{w}\right. \\
& \left.\left(\operatorname{Exp}\left(\left(\sin \frac{w}{A}\right)^{2}\right)\right) \frac{d w}{w}\right) d z
\end{aligned}
$$

provided $\operatorname{Re}(z) \geq \alpha+\eta>0$ for some constant $\eta>0$ and for all $z$ on $C$. Here $Y \leq y \leq 2 Y$ ( $Y$ is assumed to exceed a large positive constant), $B_{1}\left(s_{0}\right)$ depends on $y$,

$$
\int_{Y}^{2 Y} B_{1}\left(s_{0}\right) \frac{d y}{y}=-\int_{Y}^{2 Y} Y^{-s_{0}} \Delta\left(\frac{X}{y}\right) E(y) \frac{d y}{y}
$$

and $C$ is the union of boundedly many rectifiable curves each of bounded length all contained in a circle of bounded radius and with centre $z=0$.

REMARK. For the purposes of Theorems 2 and $3, \phi(z) z^{-1}$ can be any function analytic on $C$. But for the purposes of certain applications of theorem $4, \phi(z) z^{-1}$ will have to be replaced by $F(z) z^{-1}$ and $C$ will have to be chosen appropriately. $C_{1}$ and $\varepsilon$ may be chosen to be $A^{3}$ and $A^{-100}$ respectively and $A$ has to be large enough. $d_{0}$ can be chosen to be $A / 2$.

\section{§3. THREE FUNDAMENTAL LEMMAS.}

LEMMA 1. Let $z=x+i y$ be a complex variable with $|x| \leq \frac{1}{2}$. Then, we have

$$
(a)\left|\operatorname{Exp}\left((\sin z)^{2}\right)\right| \leq e^{\frac{1}{2}}<2 \text { for all } \mathrm{y}
$$

and

(b) if $|y| \geq 2$, we have

$$
\left|\operatorname{Exp}\left((\sin z)^{2}\right)\right| \leq e^{\frac{1}{2}}(\operatorname{Exp} \operatorname{Exp}|y|)^{-1} \leq 2(\operatorname{Exp} \operatorname{Exp}|y|)^{-1}
$$

REMARK. This is Lemma 2.1 on page 38 of $[\mathrm{KR}]_{2}$. We have retained the same notation as in this reference. Notation of Lemma 1 should not be confused with the other notation of this paper. 
A very importnat result due to H.L.Montgomery and R.C.Vaughan is the following

LEMMA 2. Suppose $R \geq 2 ; \lambda_{1}, \lambda_{2} \ldots, \lambda_{R}$ are distinct real numbers and that $\delta_{n}=\min _{m \neq n} \mid \lambda_{n}-$ $\lambda_{m} \mid$. Then

$$
\left|\sum \sum_{m \neq n} \frac{a_{m} \bar{a}_{n}}{\lambda_{m}-\lambda_{n}}\right| \leq \frac{3 \pi}{2} \sum_{n}\left|a_{n}\right|^{2} \delta_{n}^{-1}
$$

REMARK. This is Theorem 1.4.1 on page 21 of $[\mathrm{KR}]_{2}$. Again we have retained the same notation as in this reference. The notation of Lemma 2 above should not be confused with the other notation of the present paper.

As a Corollary to Lemma 2 we record

LEMMA 3. We have

$$
\int_{U}^{2 U}\left|\sum_{n=1}^{R} a_{n} \lambda_{n}^{-i t}\right|^{2} d t=U \sum_{n=1}^{R}\left|a_{n}\right|^{2}+O\left(\sum_{n=1}^{R} \lambda_{n}\left|a_{n}\right|^{2}\right)
$$

where $1 \ll \lambda_{1}, \lambda_{2}, \ldots$ are positive and increasing and $\lambda_{n+1}-\lambda_{n} \gg 1$, and $a_{1}, a_{2}, \ldots a_{R}$ are any complex numbers and further $U \geq 10$.

PROOF. Lemma 3 follows from Lemma 2 on observing that

$$
\left|\log \lambda_{n}-\log \lambda_{m}\right|^{-1} \leq\left(\lambda_{m}+\lambda_{n}\right)\left|\lambda_{m}-\lambda_{n}\right|^{-1}
$$

for all $m \neq n$.

\section{§4. PROOF OF THEOREM 1. We have}

$$
\begin{aligned}
B\left(s_{0}\right) & -\sum_{\lambda_{n} \leq y} a_{n} \lambda_{n}^{-s} \Delta\left(\frac{X}{\lambda_{n}}\right) \\
& =\int_{y}^{\infty} u^{-s_{0}} \Delta\left(\frac{X}{u}\right) d\left(\sum_{\lambda_{n} \leq u} a_{n}-D(u)\right)+\int_{y}^{\infty} u^{-s_{0}} \Delta\left(\frac{X}{u}\right) D^{\prime}(u) d u \\
& =B_{2}\left(s_{0}\right)+B_{3}\left(s_{0}\right) \text { say }
\end{aligned}
$$

(where $\left.D(u)=\frac{1}{2 \pi i} \int_{z \epsilon C} \phi(z) \frac{d z}{z}\right)$.

$$
\left.B_{2}\left(s_{0}\right)=u^{-s_{0}} \Delta\left(\frac{X}{u}\right) E(u)\right]_{y-0}^{\infty}-\int_{y}^{\infty} E(u) \frac{d}{d u}\left(u^{-s_{0}} \Delta\left(\frac{X}{u}\right)\right) d u .
$$

Here the first term is $B_{1}\left(s_{0}\right)$ which is the second term on the RHS of (8). Also the second term here is the sum of the third term and foruth term on the RHS of (8). 
Next

$$
\begin{aligned}
B_{3}\left(s_{0}\right)= & \int_{y}^{\infty} u^{-s_{0}} \Delta\left(\frac{X}{u}\right) D^{\prime}(u) d u \\
= & \int_{y}^{\infty} u^{-s_{0}}\left(\frac{1}{2 \pi i} \int_{C_{0}-i \infty}^{C_{0}+i \infty}\left(\frac{X}{u}\right)^{w}\left(\operatorname{Exp}\left(\left(\sin \frac{w}{A}\right)^{2}\right)\right) \frac{d w}{w}\right) \\
& \left(\frac{1}{2 \pi i} \int_{z \varepsilon C} u^{z-1} \phi(z) d z\right) d u \\
= & \int_{0}^{\infty} \ldots+\left(-\int_{0}^{y} \ldots\right)=B_{4}\left(s_{0}\right)+B_{5}\left(s_{0}\right), \text { say. }
\end{aligned}
$$

Now

$$
\begin{gathered}
B_{4}\left(s_{0}\right)=\int_{0}^{\infty} v^{-s_{0}} X^{-s_{0}}\left\{\left(\frac{1}{2 \pi i} \int_{C_{0}-i \infty}^{C_{0}+i \infty} v^{-w}\left(\operatorname{Exp}\left(\left(\sin \frac{w}{A}\right)^{2}\right)\right) \frac{d w}{w}\right)\right. \\
\left.\left(\frac{1}{2 \pi i} \int_{z \varepsilon C} v^{z-1} X^{z-1} \phi(z) d z\right) X\right\} d v
\end{gathered}
$$

(by the transformation $u=v X$ )

$$
\begin{aligned}
= & {\left[\frac { 1 } { 2 \pi i } \int _ { z \varepsilon C } X ^ { - s _ { 0 } + z } \left\{\frac{1}{2 \pi i} \int_{0}^{\infty} v^{-s_{0}+z-1} \int_{C_{0}-i \infty}^{C_{0}+i \infty} v^{-w}\right.\right.} \\
& \left.\left(\operatorname{Exp}\left(\left(\sin \frac{w}{A}\right)^{2}\right)\right) \frac{d w}{w} d v\right\} \phi(z) d z \\
= & \frac{1}{2 \pi i} \int_{z \varepsilon C} X^{-s_{0}+z}\left(\operatorname{Exp}\left(\left(\sin \frac{-s_{0}+z}{A}\right)^{2}\right)\right) \frac{\phi(z)}{-s_{0}+z} d z
\end{aligned}
$$

by using the fact that

$$
\begin{gathered}
\frac{1}{2 \pi i} \int_{0}^{\infty} v^{-s_{0}+z-1} \int_{C_{0}-i \infty}^{C_{0}+i \infty} v^{-w}\left(\operatorname{Exp}\left(\left(\sin \left(\frac{w}{A}\right)\right)^{2}\right) \frac{d w}{w} d v\right. \\
=\left(\operatorname{Exp}\left(\sin \left(\frac{-s_{0}+z}{A}\right)\right)^{2}\right)\left(-s_{0}+z\right)^{-1} .
\end{gathered}
$$

(We remark that under fairly general conditions on $F(w)$ we have

$$
\frac{1}{2 \pi i} \int_{0}^{\infty} x^{s-1}\left(\int_{c-i \infty}^{c+i \infty} F(w) x^{-w} d w\right) d x=F(s) .
$$

see page $7, \S 1.5$ of $[\mathrm{ECT}])$.

Finally

$$
\begin{aligned}
B_{5}\left(s_{0}\right)= & -\int_{0}^{y} u^{-s_{0}}\left\{\left(\frac{1}{2 \pi i} \int_{C_{0}-i \infty}^{C_{0}+i \infty}\left(\frac{X}{u}\right)^{w}\left(\operatorname{Exp}\left(\sin \frac{W}{A}\right)^{2}\right) \frac{d w}{w}\right)\right. \\
& \left.\left(\frac{1}{2 \pi i} \int_{z \epsilon C} u^{z-1} \phi(z) d z\right)\right\} d u
\end{aligned}
$$




$$
\begin{aligned}
= & -\frac{1}{2 \pi i} \int_{z \epsilon C} \frac{y^{-s_{0}+z}}{-s_{0}+z} \phi(z) d z \\
- & \int_{0}^{y} u^{-s_{0}}\left\{\left(\frac{1}{2 \pi i} \int_{-d_{0}-i \infty}^{-d_{0}+i \infty}\left(\frac{X}{u}\right)^{w}\left(\operatorname{Exp}\left(\sin \frac{w}{A}\right)^{2}\right) \frac{d w}{w}\right)\right. \\
& \left.\left(\frac{1}{2 \pi i} \int_{z \epsilon c} u^{z-1} \phi(z) d z\right)\right\} d u
\end{aligned}
$$

(on moving the line of integration to $\operatorname{Re}(w)=-d_{0}$ ). Clearly the last triple integral is

$$
-\frac{1}{2 \pi i} \int_{z \varepsilon C} \phi(z)\left(\frac{1}{2 \pi i} \int_{-d_{0}-i \infty}^{-d_{0}+i \infty} \frac{y^{-s_{0}+z}}{-s_{0}+z-w}\left(\frac{X}{y}\right)^{w}\left(\operatorname{Exp}\left(\sin \frac{w}{A}\right)^{2}\right) \frac{d w}{w} d z\right.
$$

and this proves Theorem 1 completely.

We next record Theorem 2 (which is a corollary to Theorem 1) and next derive two theorems (Therems 3 and 4) which follow from it.

THEOREM 2 With the same notation as in Theorem 1, and $t_{0}=Y \leq y \leq 2 Y$, we have

$$
\begin{aligned}
\mid B\left(s_{0}\right) \log 2 & -\int_{Y}^{2 Y}\left\{s_{0} \int_{y}^{\infty} E(u) u^{-s_{0}-1} \Delta\left(\frac{X}{u}\right) d u\right. \\
& \left.+\int_{y}^{\infty} E(u) u^{-s_{0}-1} J\left(\frac{X}{u}\right) d u\right\} \frac{d y}{y} \mid \\
& \leq\left|\int_{Y}^{2 Y} \sum_{\lambda_{n} \leq y} a_{n} \lambda_{n}^{-s_{0}} \Delta\left(\frac{X}{\lambda_{n}}\right) \frac{d y}{y}\right| \\
& +\left|\int_{Y}^{2 Y} y^{-s_{0}} \Delta\left(\frac{X}{y}\right) E(y) \frac{d y}{y}\right|+O\left(M^{-A}\right)+O\left(Y^{\theta-\alpha-1}\right)
\end{aligned}
$$

where $\theta=\max \operatorname{Re}(z)$ for $z \epsilon C$.

PROOF. The proof is trivial. (The last three terms on the RHS of (8) contribute the O-terms in $(14))$.

Before stating Theorem 3 we state a lemma.

LEMMA 4. Let $Y \leq y \leq 2 Y$ ( $Y$ should exceed a large positive constant), $s_{0}=\alpha+i t_{0}$ and let $\beta$ be a constant satisfying $0<\beta<1$. Then

$$
\begin{aligned}
& \int_{t_{0}^{\beta}}^{t_{0}}\left(\int_{Y}^{2_{Y}}\left|\int_{y}^{\infty} G(u) u^{-s_{0}-1} d u\right|^{2} \frac{d y}{y}\right) d t_{0} \\
& <<\int_{Y}^{\infty}\left|\frac{G(u)}{u^{\alpha}}\right|^{2} \frac{d u}{u}+t_{0} \int_{Y}^{\infty}\left|\frac{G(u)}{u^{\alpha}}\right|^{2} \frac{d u}{u^{2}}
\end{aligned}
$$


where $G(u)$ is any complex valued function of a defined in $u \geq Y$, such that the inner integral on the LHS of (15) is convergent.

PROOF. LHS of (15) is the same as

$$
\begin{aligned}
& \int_{t_{0}^{\beta}}^{t_{0}} \int_{Y}^{2 Y}\left|\int_{0}^{1} \sum_{n=1}^{\infty} G(n-1+y+u)(n-1+y+u)^{-s_{0}-1} d u\right|^{2} \frac{d y}{y} d t_{0} \\
& \leq \int_{t_{0}^{\beta}}^{t_{0}} \int_{Y}^{2 Y} \int_{0}^{1}\left|\sum_{n=1}^{\infty} G(n-1+y+u)(n-1+y+u)^{-s_{0}-1} d u\right|^{2} \frac{d y}{y} d t_{0} \\
& =\int_{Y}^{2 Y} \int_{0}^{1} \sum_{n=1}^{\infty}\left(t_{0}+O(n-1+y+u)|G(n-1+y+u)|^{2}\right. \\
& (n-1+y+u)^{-2 \alpha-2} d u \frac{d y}{y}
\end{aligned}
$$

(on reversing the order of integration and using Lemma 3)

$$
\begin{aligned}
& \ll \int_{Y}^{2 Y} \int_{0}^{1} \sum_{n=1}^{\infty}|G(n-1+y+u)|^{2}(n-1+y+u)^{-2 \alpha-2} \\
& \left(t_{0}+n-1+y+u\right) d u \frac{d y}{y} \\
= & \int_{Y}^{2 Y} \int_{y}^{\infty}\left|\frac{G(u)}{u^{\alpha}}\right|^{2} \frac{d u}{u} \frac{d y}{y}+t_{0} \int_{Y}^{2 Y} \int_{y}^{\infty}\left|\frac{G(u)}{u^{\alpha}}\right|^{2} \frac{d u}{u^{2}} \frac{d y}{y} \\
\leq & (\log 2)\left\{\int_{Y}^{\infty}\left|\frac{G(u)}{u^{\alpha}}\right|^{2} \frac{d u}{u}+t_{0} \int_{Y}^{\infty}\left|\frac{G(u)}{u^{\alpha}}\right|^{2} \frac{d u}{u^{2}}\right.
\end{aligned}
$$

This proves the Lemma.

THEOREM 3. We have

$$
\begin{aligned}
& \int_{t_{0}^{\beta}}^{t_{0}}\left|B\left(s_{0}\right)\right|^{2} \frac{d t_{0}}{t_{0}^{2}} \ll\left\{\int_{t_{0}}^{X^{4}}\left|\frac{E(u)}{u^{\alpha}}\right|^{2} \frac{d u}{u}+t_{0}^{-\beta} \int_{t_{0}^{\beta}}^{X^{4}}\left|\frac{E(u)}{u^{\alpha}}\right|^{2} \frac{d u}{u}\right. \\
& \left.+\left(\sum_{\lambda_{n} \leq 2 t_{0}}\left|a_{n}\right|^{2} \lambda_{n}^{-2 \alpha}\right) t_{0}^{-\beta}+M^{-A} t_{0}^{-\beta}+t_{0}^{(2(\theta-\alpha)-3) \beta}\right\}
\end{aligned}
$$

where $\theta=\max (\operatorname{Re}(z))$ for $z \epsilon C\left(\theta\right.$ is subject to $\left.\alpha+\frac{3}{2}-\eta \geq \theta \geq \alpha-\eta\right), \eta>0$ being a small constant and $\beta$ is a constant with $0<\beta<1$. If $\theta \geq \alpha+\frac{3}{2}$ the last term on the RHS of (16) will have to be replaced by $t_{0}^{2(\theta-\alpha)-3}$

REMARK. Note that $(16)$ holds with $B\left(s_{0}\right)$ replaced by $F\left(s_{0}\right)$. Also

$$
\sum_{I} \int_{I}\left|F\left(s_{0}\right)\right|^{2} \frac{d t_{0}}{t_{0}^{2}} \leq \int_{t_{0}^{\beta}}^{t_{0}}\left|B\left(s_{0}\right)\right|^{2} \frac{d t_{0}}{t_{0}^{2}}+M^{-A}
$$


where the I's are any set of disjoint sub-intervals of $\left[t_{0}^{\beta}, t_{0}\right]$ such that $B\left(s_{0}\right)$ is an approximation to $F\left(s_{0}\right)$.

PROOF. Note that $E(u)=O\left(u^{C_{0}+1+\theta}\right)$ and so Lemma 4 is applicable (with $Y$ replaced by $t_{0}$ after (15) is established. Note that if we replace $Y$ by $t_{0}$ before establishing (15) there is some problem in reversing the order of integration). The first term on the RHS of (15) gives the third term on the RHS of (16). The second term on the RHS of (14) gives

$$
\begin{gathered}
\int_{t_{0}^{\beta}}^{t_{0}}\left|\int_{Y}^{2 Y} y^{-s_{0}-1} \Delta\left(\frac{X}{y}\right) E(y) d y\right|^{2} d t_{0} \\
=\int_{t_{0}^{\beta}}^{t_{0}}\left|\int_{Y}^{\infty} \ldots-\int_{2 Y}^{\infty} \cdots\right|^{2}<<\int_{t_{0}}^{\infty}\left|\frac{E(u)}{u^{\alpha}}\right|^{2}\left|\Delta\left(\frac{X}{u}\right)\right|^{2} \frac{d u}{u},
\end{gathered}
$$

remembering our choice $Y=t_{0}$. Note that the contribution to the last integral from $u \geq X^{4}$ is $O\left(M^{-A}\right)$.

Similarly (and in a simpler way) we obtain the following Theorem from Theorem 1 (Putting $Y=t_{0}$ in (9)).

THEOREM 4. We have

$$
\begin{aligned}
& \left|B\left(s_{0}\right)\right| \ll\left\{t_{0} \int_{t_{0}}^{X^{4}}\left|\frac{E(u)}{u^{\alpha}}\right| \frac{d u}{u}+\sum_{\lambda_{n} \leq 2 t_{0}}\left|a_{n}\right| \lambda_{n}^{-\alpha}\right. \\
& \left.+M^{-A}+t_{0}^{\theta-\alpha-1}\right\}
\end{aligned}
$$

and so

$$
\begin{aligned}
& t_{0}^{-1}\left|B\left(s_{0}\right)\right| \ll\left\{\int_{t_{0}}^{X^{4}}\left|\frac{E(u)}{u^{\alpha}}\right| \frac{d u}{u}+\left(\sum_{\lambda_{n} \leq 2 t_{0}}\left|a_{n}\right| \lambda_{n}^{-\alpha}\right) t_{0}^{-1}\right. \\
& \left.+t_{0}^{-1} M^{-A}+t_{0}^{\theta-\alpha-2}\right\} .
\end{aligned}
$$

REMARK. Some times it is more advantageous to use lower bounds for

$$
\int_{t_{0}}^{X^{4}}\left|\frac{E(u)}{u^{\alpha}}\right|^{2} \frac{d u}{u}
$$

and insert an upper bound for $E(u)$ say $O\left(u^{\alpha_{0}}\right)\left(\alpha_{0}<2 \alpha\right)$ and thus obtain a lower bound for

$$
\int_{t_{0}}^{X^{4}}\left|\frac{E(u)}{u^{2 \alpha-\alpha_{0}}}\right| \frac{d u}{u}
$$

of the type $\gg \log t_{0}$ (a weaker lower bound will result in a weaker $\Omega_{ \pm}$result). We can use such a lower bound to prove $E(u)=\Omega_{ \pm}\left(u^{2 \alpha-\alpha_{0}}\right)$ under fairly general (but not too general) conditions on $F(s)$. We will amplify this remak later. 


\section{§5. APPLICATIONS OF THEOREMS 3 AND 4 TO $\Omega$ THEOREMS.}

From now on we assume the convergence of $\sum\left|a_{n}\right| \lambda_{n}^{-\sigma}$ for every $\sigma>1$. (In applications of Theorem 3 we need the convergence of $\sum\left|a_{n}\right|^{2} \lambda^{-\sigma}$ for every $\sigma>1$. Of course the convergence of $\sum\left|a_{n}\right| \lambda_{n}^{-\sigma}$ for every $\sigma>1$ is a consequence). Naturally $F(s)$ will be regular in $\sigma>1$ with the consequence $\theta \leq 1+\varepsilon$ for every fixed $\varepsilon>0$. We choose $\beta(<1)$ close to 1 and $\alpha(1 \geq \alpha-\eta>0)$ ( $\eta$ is a small positive constant) and $\left\{t_{0}\right\}$ a sequence which tends to infinity for which LHS of (16) is $\gg 1$ (It will be $\gg \log t_{0}$ or a bigger quantity in some applications). We give some details of application to $\Omega$ theorems in the case of $F(s)=(\zeta(s))^{k}$ and $F(s)=\operatorname{Exp}(\zeta(s)$ ). (We note that a lower bound for

$$
\sum_{I} \int_{I}\left|B\left(s_{0}\right)\right|^{2} \frac{d t_{0}}{t_{0}^{2}}
$$

I running through some disjoint sub-intervals of $\left[t_{0}^{\beta}, t_{0}\right]$ wherein $B\left(s_{0}\right)$ approximates to $F\left(s_{0}\right)$ with a small error is enough for many purposes). The remark in the parenthesis is applicable to $F(s)$ with many singularities but which are not too densely distributed, (see [RB, KR, MVS]). For example it is applicable to $\operatorname{Exp} \operatorname{Exp}\left((\zeta(s))^{\frac{1}{2}}\right)$. If $F(s)$ has no singularities in $(\sigma \geq$ $\left.\alpha-\delta, t_{0}^{\beta} \leq t \leq t_{0}\right), B(s)$ approximates to $F(s)$ throughout the interval

$$
t_{0}^{\beta}+C_{1} \log \log M, t_{0}-C_{1} \log \log M
$$

and hence it suffices to consider a lower bound for

$$
\int_{t_{0}^{\beta}}^{t_{0}}\left|B\left(s_{0}\right)\right|^{2} \frac{d t_{0}}{t_{0}^{2}}
$$

or equivalently for

$$
\int_{t_{0}^{\beta}}^{t_{0}}\left|F\left(s_{0}\right)\right|^{2} \frac{d t_{0}}{t_{0}^{2}}
$$

LEMMA 5. We have for all $T \geq 10$ and all integers $k \geq 2$,

$$
\frac{1}{T} \int_{T}^{2 T}\left|\zeta\left(\frac{1}{2}-\frac{1}{2 k}+i t\right)\right|^{2} d t \gg T^{\frac{1}{k}}
$$

and by Hölders inequality

$$
\frac{1}{T} \int_{T}^{2 T}\left|\zeta\left(\frac{1}{2}-\frac{1}{2 k}+i t\right)\right|^{2 k} \gg T
$$

and so

$$
\int_{T^{\beta}}^{T}\left|\zeta\left(\frac{1}{2}-\frac{1}{2 k}+i t\right)\right|^{2 k} \frac{d t}{t^{2}} \gg \log T .
$$

REMARK. The proof of (2) does not need the functional equation. It needs (apart from Lemma 3) the result

$$
\zeta(s)=\sum_{n \leq 10 T} n^{-s}+O\left(T^{-\sigma}\right)
$$


(Note that $\frac{1}{T} \int_{T}^{2 T}|\zeta(\sigma+i t)|^{2} d t=O\left(T^{1-2 \sigma}\right)$ for all $\sigma<\frac{1}{2}$ and $\gg(1-2 \sigma)^{-1} T^{1-2 \sigma}$ for all $\sigma<\frac{1}{2}$ and close to $\frac{1}{2}$. Convexity now gives the mean value to be $\gg T^{1-2 \sigma}$ for all $\sigma<\frac{1}{2}$.) uniformly in $(\sigma \geq 0, T \leq t \leq 2 T)$ (see $[\mathrm{KR}]_{1}$ or the Third main theorem of Chapter II in $[\mathrm{KR}]_{2}$. These do not depend upon the functional equation. The result in $[\mathrm{KR}]_{2}$ is very very general and very very important from many points of view). Next we remark that Theorem 3 together with $(22)$ gives

THEOREM 5. We have

$$
\sum_{n \leq x} d_{k}(n)-x P_{k}(\log x)=\Omega\left(x^{\frac{1}{2}-\frac{1}{2 k}}\right)
$$

where $(\zeta(s))^{k}=\sum_{n=1}^{\infty} d_{k}(n) n^{-s}(s=\sigma+i t, \sigma \geq 2)$ and $k(\geq 2)$ is any integer and $P_{k}(\log x)$ is a polynomial of degree $k-1$ in $(\log x)$.

REMARK. All our results $\Omega\left(N_{1}\right)$ and $\Omega_{ \pm}\left(N_{2}\right)$ are effective in the following sense. Given any $A>100$, we can find a $B(>A)$ effectively such that

$$
\begin{gathered}
\max _{A \leq x \leq B}|E(x)|>\kappa_{1} N_{1} \\
\max _{A \leq x \leq B} E(x)>\kappa_{2} N_{2} \\
\min _{A \leq x \leq B} E(x)<-\kappa_{3} N_{2}
\end{gathered}
$$

where $E(x)$ is the error function and $\kappa_{1}$ and $\kappa_{2}$ and $\kappa_{3}$ are effective positive constants (independent of $N_{1}$ and $N_{2}$ ).

In our applications to say $\operatorname{Exp}(\zeta(s))$ and so on, an important role is played by BorelCatheodory theorem (see $\S 1.6$, p. 26 of $[\mathrm{KR}]_{2}$ ) which we state as

LEMMA 6. Suppose $f(z)$ is analytic in $\left|z-z_{0}\right| \leq R$, and on the circle $z=z_{0}+R e^{i \theta}$ (0 $\leq$ $\theta \leq 2 \pi)$ we have $\operatorname{Re}(f(z)) \leq U$. Then in $\left|z-z_{0}\right| \leq r<R$, we have

$$
\left|f(z)-f\left(z_{0}\right)\right| \leq \frac{2 r\left(U-\operatorname{Re}\left(f\left(z_{0}\right)\right)\right)}{R-r}
$$

and so

$$
|f(z)| \leq \frac{2 r U}{R-r}+\frac{R+r}{R-r}\left|f\left(z_{0}\right)\right| . .
$$

As a corollary we deduce 
LEMMA 7. Let $z_{0}=2+i t_{0}, R=1+2 \varepsilon, r=1+\varepsilon$ where $\varepsilon>0$ is a very small constant. Let $g(s)$ be analytic in $\left|z-z_{0}\right| \leq R$. Let $H\left(t_{0}\right)$ be positive and tend to infinity as $t_{0} \rightarrow \infty$. Let $\left|g\left(z_{0}\right)\right|$ be bounded by an absolute constant and let

$$
\max _{\left|s-z_{0}\right| \leq r}|g(s)| \geq H\left(t_{0}\right)
$$

Then

$$
\max _{\left|s-z_{0}\right|=R} \operatorname{Re}(g(s)) \geq\left(H\left(t_{0}\right)\right)^{1-\varepsilon}
$$

for every $\varepsilon>0$ and all $t_{0}$ exceeding a large positive constant.

PROOF. Assume that (26) is false. Then by Lemma 6, we have

$$
\max _{\left|s-z_{0}\right| \leq r}|g(s)| \leq \frac{2(1+2 \varepsilon)}{\varepsilon}\left(H\left(t_{0}\right)\right)^{1-\varepsilon}+\frac{2+3 \varepsilon}{\varepsilon}\left|g\left(z_{0}\right)\right|
$$

which contradicts (25). Hence Lemma 7 is proved.

LEMMA 8. We have

$$
\max _{\left|s-z_{0}\right|=R}|\operatorname{Exp}(\zeta(s))| \geq \operatorname{Exp} \operatorname{Exp}\left(\left(\log t_{0}\right)^{1-3 \varepsilon}\right)
$$

and also

$$
\max _{\left|s-z_{0}\right|=R}|\operatorname{Exp} \operatorname{Exp}(\zeta(s))| \geq \operatorname{Exp} \operatorname{Exp} \operatorname{Exp}\left(\left(\log t_{0}\right)^{1-4 \varepsilon}\right)
$$

for every fixed $\varepsilon>0$.

REMARK. By convexity (coupled with the kernel $\operatorname{Exp}\left((\sin z)^{2}\right)(27)$ and (28) hold for $s=\alpha+i t$, where $\left|t_{1}-t_{0}\right| \leq \frac{1}{2} t_{0}$ and $\alpha(<1)$ is as close to 1 as we want.

PROOF. To prove (27) we should have

$$
\max _{\left|s-z_{0}\right|=R}\left(R e(\zeta(s))>\operatorname{Exp}\left(\left(\log t_{0}\right)^{1-3 \varepsilon}\right)\right.
$$

But this follows from

$$
\max _{\left|s-z_{0}\right| \leq r}|\zeta(s)|>\operatorname{Exp}\left(\left(\log t_{0}\right)^{1-\varepsilon}\right) .
$$

(See Theorems 3.3.1 and 3.3.2 of $[\mathrm{KR}]_{2}$. In fact there exist plenty of such $t_{0}$ ). The result (28) can also be proved similarly (using (27)).

REMARK. We can prove

$$
\max _{\left|s-z_{0}\right| \leq r} \mid \operatorname{Exp} \operatorname{Exp} \operatorname{Exp}\left((\zeta(s)) \mid>\operatorname{Exp} \operatorname{Exp} \operatorname{Exp} \operatorname{Exp}\left(\left(\log t_{0}\right)^{1-4 \varepsilon}\right)\right.
$$


and so on. But we are unable to prove $\Omega$ for results for the error terms in the asymptotic formula for the summatory function of the coefficients for such (and higher) iterates of Exp. Aslo while dealing with $\operatorname{Exp} \operatorname{Exp}\left((\zeta(s))^{\frac{1}{2}}\right.$ ) (we can certainly deal with $\Omega$ theorem for such functions) we have to use density theorems for the zeros of the Riemann zeta-function.

Theorem 4 together with Lemma 8 gives

THEOREM 6. Let

$$
\left.\begin{array}{l}
\operatorname{Exp}\left((\zeta(s))=\sum_{n=1}^{\infty} b_{n} n^{-s}\right. \text { and } \\
\operatorname{Exp} \operatorname{Exp}\left((\zeta(s))=\sum_{n=y}^{\infty} d_{n} n^{-s}\right.
\end{array}\right\}
$$

in $\operatorname{Re}(s)>1$. Then, we have

$$
\sum_{n \leq x} b_{n}=\frac{1}{2 \pi i} \int_{|s-1|=\frac{1}{10}} \operatorname{Exp}(\zeta(s)) x^{s} \frac{d s}{s}+\Omega\left(x^{1-\varepsilon}\right)
$$

and

$$
\sum_{n \leq x} d_{n}=\frac{1}{2 \pi i} \int_{|s-1|=\frac{1}{10}} \operatorname{Exp} \operatorname{Exp}(\zeta(s)) x^{s} \frac{d s}{s}+\Omega\left(x^{1-\varepsilon}\right)
$$

hold good for every fixed $\varepsilon>0$ as $x \rightarrow \infty$. In fact if $\alpha$ is any constant such that $\frac{99}{100}<\alpha<1-\eta$ holds for some $\eta>0$ and if $E(x)$ denotes either error term, we have

$$
t_{0}^{-1} R\left(t_{0}\right) \ll \int_{t_{0}}^{X^{4}}\left|\frac{E(u)}{u^{\alpha}}\right| \frac{d u}{u}
$$

where $R\left(t_{0}\right)$ denotes the RHS of (27) and (28) respectively.

REMARK. Our method of proving (33) is applicable to functions like $\operatorname{Exp}\left((\zeta(s))^{\frac{1}{2}}\right)$ and also to $\operatorname{Exp} \operatorname{Exp}\left((\zeta(s))^{\frac{1}{2}}\right)$. As remarked earlier we have to use the density theorems for the zeros of the Riemann zeta-function. In such cases the dominant term is

$$
\frac{1}{2 \pi i} \int_{-\infty}^{0+}(\text { generating function at } \mathrm{s}+1) \frac{\mathrm{x}^{\mathrm{s}+1}}{\mathrm{~s}+1} \mathrm{ds}
$$

(with the commonly employed notation). Our results are applicable to functions like $L(s, \chi)$ (and so on) in place of $\zeta(s)$ ). But we are unable to handle $\Omega_{ \pm}$results except under stringent conditons to be stated in the next section.

\section{$\S 6 . A P P L I C A T I O N S$ OF THEOREMS 3 AND 4 TO $\Omega_{ \pm}$THEOREMS.}




\section{THEOREM 7. Let}

$$
F(s)=\sum_{n=1}^{\infty} a_{n} \lambda_{n}^{-s}(s=\sigma+i t)
$$

where $a_{n}^{\prime}$ s are real numbers and $1 \ll \lambda_{1}<\lambda_{2}<\ldots, \lambda_{n+1}-\lambda_{n} \gg 1$. We assume that $F(s)$ is absolutely convergent in $\sigma>1$ and is analytically continuable in $(\sigma \geq \alpha-\delta$ (where $0<\delta<\alpha<1, \delta$ and $\alpha$ being constants), $t \geq C_{2}$ where $C_{2}$ is some large constant). In this region we assume

$$
|F(s)|<t^{C_{3}}
$$

where $C_{3}$ is a positive constant which may depend on $\alpha$ and $\delta$. Lastly we assume (for every $\left.T \geq C_{2}\right)$

$$
\int_{U}^{2 U}\left|\frac{E(u)}{u^{\alpha}}\right| \frac{d u}{u}>C_{4}
$$

for some $U\left(T \leq U \leq T^{C_{5}}\right)$ for some positive constants $C_{4}$ and $C_{5}$. Here

$$
E(u)=\sum_{\lambda_{n} \leq u} a_{n}-\frac{1}{2 \pi i} \int_{C} F(s) u^{s} \frac{d s}{s}
$$

$C$ being a curve joining $\alpha-\delta-i C_{2}, 1+\varepsilon-i C_{2}, 1+\varepsilon+i C_{2}$ and $\alpha-\delta+i C_{2}$ in this order ( $\varepsilon>0$ being any small constant). Then as $x \rightarrow \infty$, there holds

$$
E(x)=\Omega_{ \pm}\left(x^{\alpha}\right)
$$

Proof. The proof is nearly the same as our earlier one (see [AS, KS] for a nice exposition in the case $F(s)=\zeta(s) \zeta(2 s) \zeta(3 s) \ldots)$.

COROLLARY 1. Let $\gamma$ be the Euler's constant and $E(x)=\sum_{n \leq x} d(n)-(x \log x+(2 \gamma-1) x)$. Then

$$
E(x)=\Omega_{ \pm}\left(x^{\frac{1}{6}}\right)
$$

REMARK. We use

$$
\int_{U}^{2 U}\left|\frac{E(u)}{u^{\frac{1}{4}}}\right|^{2} \frac{d u}{u} \gg 1
$$

which follows from theorem 3 (in the special case $\left.F(s)=(\zeta(s))^{2}\right)$. From this we deduce

$$
\int_{U}^{2 U}\left|\frac{E(u)}{u^{\frac{1}{6}}}\right| \frac{d u}{u} \gg 1
$$

using the estimate $E(u)=O\left(u^{\frac{1}{3}}\right)$ due to G.Voronoi (see p.272 of [GHH, EMW]). Our results are applicable to circle problem where the generating function is $F(s)=\zeta(s) L(s, \chi))$ for the nontrivial character $\chi(\bmod 4)$. 
COROLLARY 2. Let $F(s)=\prod_{k=1}^{\infty} \zeta(k s)$. Then the dominant term is $\sum_{j=1}^{10} C_{j} x^{\frac{1}{j}}$ (where $C_{1}, C_{2}, \ldots, C_{10}$ are suitable real constants). With $\alpha=\frac{1}{10}$ we obtain

$$
E(x)=\Omega_{ \pm}\left(x^{\frac{1}{10}} \operatorname{Exp}\left(C_{11}\left(\frac{\log x}{\log \log x}\right)^{\frac{1}{2}}\right)\right)
$$

where $C_{11}>0$ is a certain constant.

REMARK. Here instead of using

$$
\int_{U}^{2 U}\left|\frac{E(u)}{u^{\frac{1}{6}}}\right|^{2} \frac{d u}{u} \gg \log T
$$

we find it advantageous to apply Theorem 4 directly and obtain

$$
\int_{U}^{2 U}\left|\frac{E(u)}{u^{\frac{1}{10}}}\right| \frac{d u}{u} \gg \operatorname{Exp}\left(C_{12}\left(\frac{\log T}{\log \log T}\right)^{\frac{1}{2}}\right) .
$$

and deduce the $\Omega_{ \pm}$theorem from this.

FINAL REMARK. The results on circle and divisor problems are not new. There are very classical and better results due to G.H.Hardy, E.Landau and others. We have not given references to these classical results. In recent times these have been improved by Raghavan Narasimhan, K.Chandrasekharan, K.S.Gangadharan, K.A.Corradi, I Katai, J.L.Halfner and others. We hope that the reader will be able to find out references to these famous papers. 


\section{References}

[RB,KR], R.BALASUBRAMANIAN and K.RAMACHANDRA, Some problems of analytic number theory-III, HARDY-RAMANUJAN J., Vol 4(1981), 13-40.

[RB, KR, MVS], R.BALASUBRAMANIAN, K.RAMACHANDRA and M.V.SUBBARAO, On the error function in the asymptotic formula for the counting function of $k$-full numbers. Acta Arith, 50(1988), 107-118.

[GHH, EMW], G.H.HARDY and E.M.WRIGHT, Introduction to the theory of numbers (fifth edition) Oxford Science publications (1979).

$[\mathrm{KR}]_{1}$ K.RAMACHANDRA, Riemann zeta-fuction, Ramanujan Institute, Madras University, Madras (1979), 16 pp.

$[\mathrm{KR}]_{2}$ K.RAMACHANDRA, On the mean-value and Omega-theorems for the Riemann zetafunction, Lecture notes no. 85, TIFR (Narosa (Springer Verlag)) 1995.

[AS], A. SCHINZEL, On an analytic problem considered by Sierpinski and Ramanujan. New Trends in probability and Statistics, 2(Palanga 1991), 165-171, VSP, UTRECHT (1992).

[AS, KS], A.SANKARANARAYANAN and K.SRINIVAS, On a method of Balasubramanian and Ramachandra (On the abelian group problem), Rend.Sem.Mat. Univ. Padova Vol. 97(1997), 135-161.

[ECT] E.C.TITCHAMARSH, Introduction to the Theory of Foruier integrals, Oxford University Press, Ely house, London W.1, Seventh edn. (1975) First edn. (1937)) 


\section{$\underline{\text { ACKNOWLEDGMENTS }}$}

The authors are very much thankful to the referee for some helpful comments during revision. The second author was a fellow of BRNS(DAE) INDIA, during the preparation of this paper and wishes to thank the organisation for its hlep to him. The authors are thankful to Professor Roger Heath-Brown FRS, for encouragment. Finally they are thankful to Smt.J.N.Sandhya for technical help. 


\section{ADDRESS OF THE AUTHORS}

1. R.Balasubramanian

Senior Professor and Director

Matscience

Tharamani P.O.

CHENNAI-600 113

INDIA.

e-mail balu@imsc.ernet.in

2. K.Ramachandra

Hon. Vis. Professor

National Institute of Advanced Studies

Indian Institute of Science Campus

Bangalore-560012

INDIA.
Also
Retd. Sr. Professor,
T I F R. Centre
P.O. Box 1234
Bangalore-560 012
INDIA.

e-mail kram@math.tifrbng.res.in

MANUSCRIPT COMPLETED ON 06-08-2002. 